\title{
The yield of sonication fluid culture for presumed aseptic loosening of orthopedic devices: a meta-analysis
}

\author{
Qingyu Zhang ${ }^{1}$, Yongming $\mathrm{Xi}^{2}$, Dong Li ${ }^{1}$, Zenong Yuan ${ }^{1}$, Jun Dong ${ }^{1}$ \\ ${ }^{1}$ Department of Orthopedics, Shandong Provincial Hospital Affiliated to Shandong First Medical University, Jinan, China; ${ }^{2}$ Department of Spine \\ Surgery, Affiliated Hospital of Qingdao University, Qingdao, China \\ Contributions: (I) Conception and design: Z Yuan, J Dong; (II) Administrative support: Y Xi, L Dong; (III) Provision of study materials or patients: Q \\ Zhang, Y Xi; (IV) Collection and assembly of data: Q Zhang, D Li; (V) Data analysis and interpretation: Q Zhang, D Li; (VI) Manuscript writing: All \\ authors; (VII) Final approval of manuscript: All authors. \\ Correspondence to: Jun Dong, MD; Zenong Yuan, MD. Department of Orthopedics, Shandong Provincial Hospital Affiliated to Shandong First \\ Medical University, No. 324, Road Jing Wu Wei Qi, Jinan 250021, China. Email: slyydj2019@163.com; Yuanzenong@126.com.
}

Backgrounda Sonication fluid culture is routinely conducted in patients with suspected orthopedic devicerelated infection (ORI). However, its value in the assessment of orthopedic devices that have explanted because of presumed aseptic loosening is still debatable. A meta-analysis was conducted to estimate the yield of sonication fluid culture in detecting the presence of microorganisms in orthopedic devices with presumed aseptic loosening, and to summarize the clinical characteristics and outcomes of these patients.

Methods: A meta-analysis was performed of peer-reviewed publications detailing the characteristics of patients whose orthopedic devices were explanted for presumed aseptic loosening and who underwent subsequent sonication fluid culture. Diagnostic data were extracted and pooled to estimate the yield of sonication fluid culture for detecting the presence of microorganisms with $95 \%$ confidence intervals (CI).

Results: Eight studies involving 421 patients were included in the meta-analysis analysis. The pooled results showed that sonication fluid culture had a yield of approximately 30\% (95\% CI: 20-41\%) for identifying the presence of microorganisms in presumed aseptic loosening of orthopedic devices; this yield was higher than that of intraoperative tissue culture (22\%, 95\% CI: 8-41\%) and aspirated fluid culture (13\%, 95\% CI: 7-21\%). Sonication and vortexing for $\leq 5 \mathrm{~min}$ had a detection rate of $49 \%$ (95\% CI: 43-55\%), which exceeded that of $>5 \mathrm{~min}$. Coagulase-negative Streptococcus accounted for 74\% (95\% CI: 51-92\%) of the isolated microorganisms. However, patients with positive microbiological results of sonication fluid culture and those with negative results showed similar outcomes after revision surgery.

Conclusions: Colonization of low-virulent microorganisms may exist in a considerable number of patients with clinically presumed aseptic loosening of orthopedic devices. When this occurs, sonication (1-5 min at $40 \mathrm{kHz}$ ) of fluid culture could act as a sensitive diagnostic tool. However, the question of whether sonication fluid culture should be integrated into the routine treatment of loosened orthopedic devices requires further investigation.

Keywords: Sonication fluid culture; aseptic loosening; orthopedic devices; meta-analysis; microorganism colonization

Submitted Jun 07, 2020. Accepted for publication Oct 30, 2020.

doi: 10.21037/apm-20-1228

View this article at: http://dx.doi.org/10.21037/apm-20-1228

^ ORCID: 0000-0002-0497-3630. 


\section{Introduction}

Aseptic loosening is a devastating complication following the implantation of orthopedic devices. As the main reason for the failure of total hip arthroplasty and spinal fusion, aseptic loosening is also the third most common cause of revised total knee arthroplasty (1-3). The occurrence of aseptic loosening can be related to factors including stress shielding, microfracture, excessive loading, sagittal imbalance, allergic reactions, poor component positioning, local strains on the implant/bone interface, obesity and the presence of wear debris (1-4). However, implantable devices are also highly susceptible to bacterial colonization, and it is likely that the incidence of infection is underestimated $(5,6)$. Many cases of presumed aseptic loosening may actually involve the presence of biofilm, especially when the typical features of infection, such as sinus tracts, highly elevated inflammatory biomarkers, local swelling, and fever, are not present $(5,6)$. Biofilms are highly structured matrices of extracellular polymeric substances built by a single or a community of bacterial species (Figure 1) (7) that support the proliferation of microorganisms on implants but no in the surrounding soft tissue. They provide microorganisms with increased protection from antibiotics and host defense mechanisms, and hamper the power of intraoperative tissue culture or aspirated fluid examinations to detect viable bacteria (8-10). The accurate identification of biofilmembedded bacteria in patients with apparent aseptic loosening is not only of medico-legal importance but also facilitates the early initiation of an appropriate therapeutic regimen by averting the placement of a new implant into an infected environment (11).

The identification of orthopedic device-related infections (ORIs) has been hindered by a lack of clear and standardized diagnostic criteria (12). Currently, cultivation techniques are the most widely used reference standard for confirming the microbial causal factors of periprosthetic joint infection (PJI), fracture-related infection (FRI), and spinal instrumentation infection in clinical practice (13-16). However, the yield of peri-implant soft tissue culture is highly dependent on sampling technique and might be compromised by the formation of microbial biofilm $(8,9)$. The use of low-frequency ultrasound (sonication) on removed implants disrupts the bacterial biofilm layer, dislodges adherent bacteria, and preserves the viability of microorganisms (17). Subsequent fluid culture or polymerase chain reaction (PCR) examination of microorganism-specific RNA is an alternative method

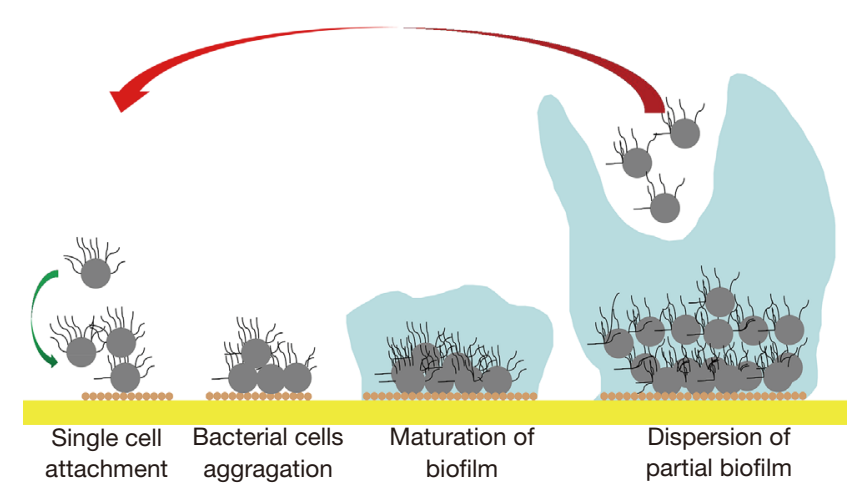

Figure 1 Schematic overview of the formation of biofilm, which can be divided into five phases. During the first phase, planktonic bacteria attach to the surface of implants and this process is reversible. The second phase, in which bacteria attach to the surface of implants and other bacterial cells, is irreversible. In the next two phases, a matrix of extracellular polysaccharide substances (EPS) is produced, and as the bacterial colonies start to grow to their maximum extent, the biofilm becomes mature and stabilized. From the second to the fourth period, embedded bacteria have no mobility. In the final phase of this cycle, bacteria inside the biofilm regain their mobility and starts to disperse to colonize at new surfaces.

for the identification of ORI (17). According to previous studies, the sensitivity and specificity of sonication fluid culture in diagnosing PJI and postoperative spinal infection are significantly elevated compared to those of conventional periprosthetic soft tissue cultures (18). Therefore, sonication can be applied in the diagnosis of low-suspicion patients with low virulent infections or highly suspected patients for whom the causative microorganism cannot be determined (18).

Inspired by these findings, in 2002 Nguyen et al. (19) first adopted sonication fluid culture to detect the presence of microorganisms in cases with presumed aseptic loosening of artificial joints. Surprisingly, they found that 1 of 21 cases initially regarded as aseptic loosening were associated with underlying subclinical infection (19). Subsequently, several studies were undertaken using sonication fluid culture to investigate various orthopedic devices (e.g., artificial joints, pedicle screws, and osteo-synthetic instruments) that had been explanted for reasons other than infection. However, the positive yield rates reported in these studies were rather heterogeneous (20-23).

In this study we systematically retrieved related 
investigations with the aims of (I) further evaluating the yield of sonication fluid culture for detecting the presence of microorganisms in presumed aseptic loosening by performing using a meta-analysis of sensitivity, (II) comparing the test power of sonication fluid culture with intra-operative culture and aspirated fluid culture; and (III) evaluating the clinical characteristics and outcomes of patients with unexpected positive culture results. The following article is presented in accordance with the PRISMA reporting checklist (available at http://dx.doi. org/10.21037/apm-20-1228) (24).

\section{Methods}

This article was registered in PROSPERO (ID CRD42020166753). As all data used were retrieved from published articles, neither ethical approval nor informed consent was required. Database searching, eligibility assessment, data extraction, and methodological quality evaluation were performed by two independent investigators (Q Zhang and D Li). Disagreement was resolved by consensus .

\section{Eligibility criteria}

Eligible studies were peer-reviewed publications containing empiric data on the yield of sonication fluid culture for human patients whose orthopedic devices (artificial joints, osteo-synthetic materials, pedicle screws) were removed for loosening without evidence of infection such as sinus tract, highly elevated serum biomarkers, local swelling and fever. Reported data were considered sufficient to extract the number of positive events and enrolled participants.

\section{Databases searching}

Searches of three electronic databases (PubMed, Embase, and the Cochrane Central Register of Controlled Trials) were conducted using a combination of the two keywords, "sonication" and "loosening". The search was open to articles from database inception to May 10, 2020 and there were no language restrictions.. The titles and abstracts of retrieved records were first screened to exclude studies that did not match the inclusion criteria. The full-texts of the remaining records and additional articles identified from the reference lists of the retrieved literature were then acquired and scrutinized for eligibility.

\section{Data collection process}

Extracted data used for quantitative analysis included the number of enrolled patients, diagnostic tools, positives cases with different detection methods, and where possible, the species of the identified pathogen. Other requisite data recorded to standardized excel files included the surname of the first author, year and region of publication, study design, inclusion interval and inclusion criteria, patient demographic characteristics (e.g., sex, age, and comorbidities), the type of orthopedic devices, and the time between index surgery and revision.

In cases of overlapping patient datasets between separate articles, the most recent or comprehensive series was selected for the meta-analysis.

\section{Risk of bias of individual studies}

The risk of bias of each study was assessed using the Quality Assessment of Diagnostic Accuracy Studies 2 (QUADAS-2) tool (24), which consists of four domains (patient selection, index test, reference standard, and flow and timing). Applicability regarding the four domains was also rated.

\section{Statistical analysis}

Statistical analysis was performed in R 3.6.1 (http://www. r-project.org/) by using the "metaprop" and "forest" functions of the "meta" package (25), which generated the proportion of the yield (true-positive) and associated 95\% confidence intervals $(\mathrm{CI})$ with an inverse variance method after logit transformation. The random-effects model of meta-analysis was adopted for the results reported in this paper. Heterogeneity across studies was assessed by using the $\mathrm{I}^{2}$ statistics, with values of $\geq 50 \%$ regarded as significant.

\section{Results}

\section{Literature search results}

The literature search identified 51 records, with 22 additional citations retrieved through scrutinizing the reference lists of related articles. After the initial screening of titles and abstracts, the full texts of 34 articles were downloaded and assessed for eligibility. Ultimately, nine (19-23,26-29) articles satisfied all the inclusion criteria for the systemic review. Bereza et al. published two $(22,29)$ studies in which overlapping patients were subject to 


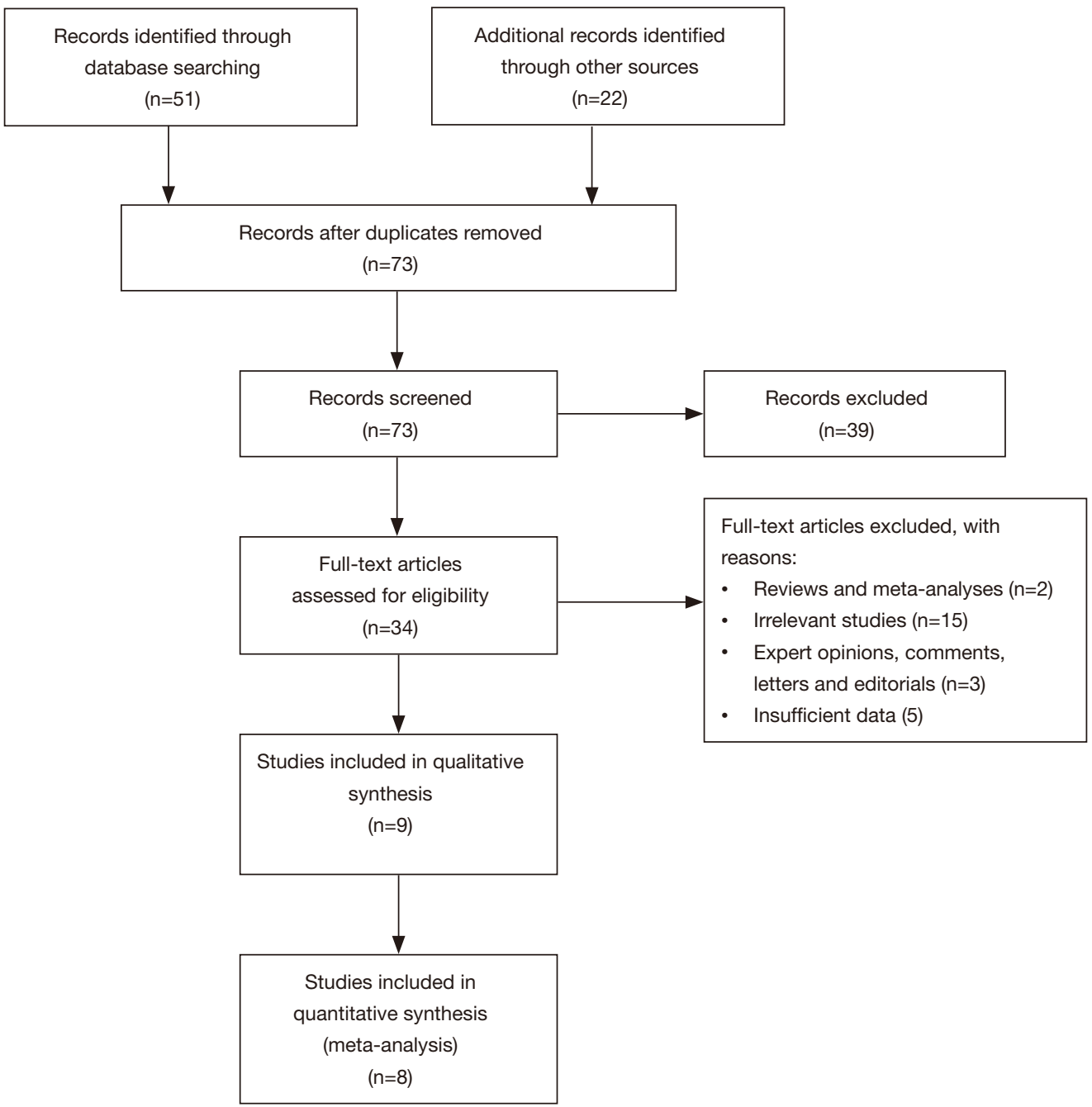

Figure 2 The selection process of the included studies.

separate microbiologic tests (sonication fluid culture and sonication fluid PCR, respectively). Because this study mainly aimed at the yield of sonication fluid culture of explanted orthopedic devices, the former (22) was analyzed quantitatively and the latter (29) qualitatively. The selection process for the included studies is outlined in Figure 2.

\section{Study population and setting}

In total, 421 patients with explanted orthopedic devices were included in this meta-analysis. Aseptic loosening was presumed in two $(20,21)$ studies investigating spinal fusion instrumentations and seven $(19,22,23,26-29)$ investigating artificial joints. The diagnosis of occult ORI or colonization established by sonication fluid culture was discussed in eight
(19-23,26-28) studies. Three $(22,26,28)$ studies also reported the result of intraoperative soft tissue culture and two $(22,28)$ described aspirated joint fluid culture. The period between the index operation and revision surgery ranged from 20.2-30.9 months for spine surgery and 79-122.8 months for arthroplasty. Figure 3 shows the patient exclusion criteria of the included studies. In four $(19,21,26,28)$ studies, aseptic loosening was defined only on the basis of clinical evidence. Patients with an elevated level of laboratory inflammatory markers were excluded in three $(20,23,27)$ studies, and two $(20,22)$ studies ruled out those with long-term antibiotic administration before surgery. The characteristics of the individual studies are presented in Table 1 . The frequency of sonication used ranged from 40 to $67 \mathrm{kHz}$, and the duration of the sonication procedure ranged from 1 to 30 minutes 


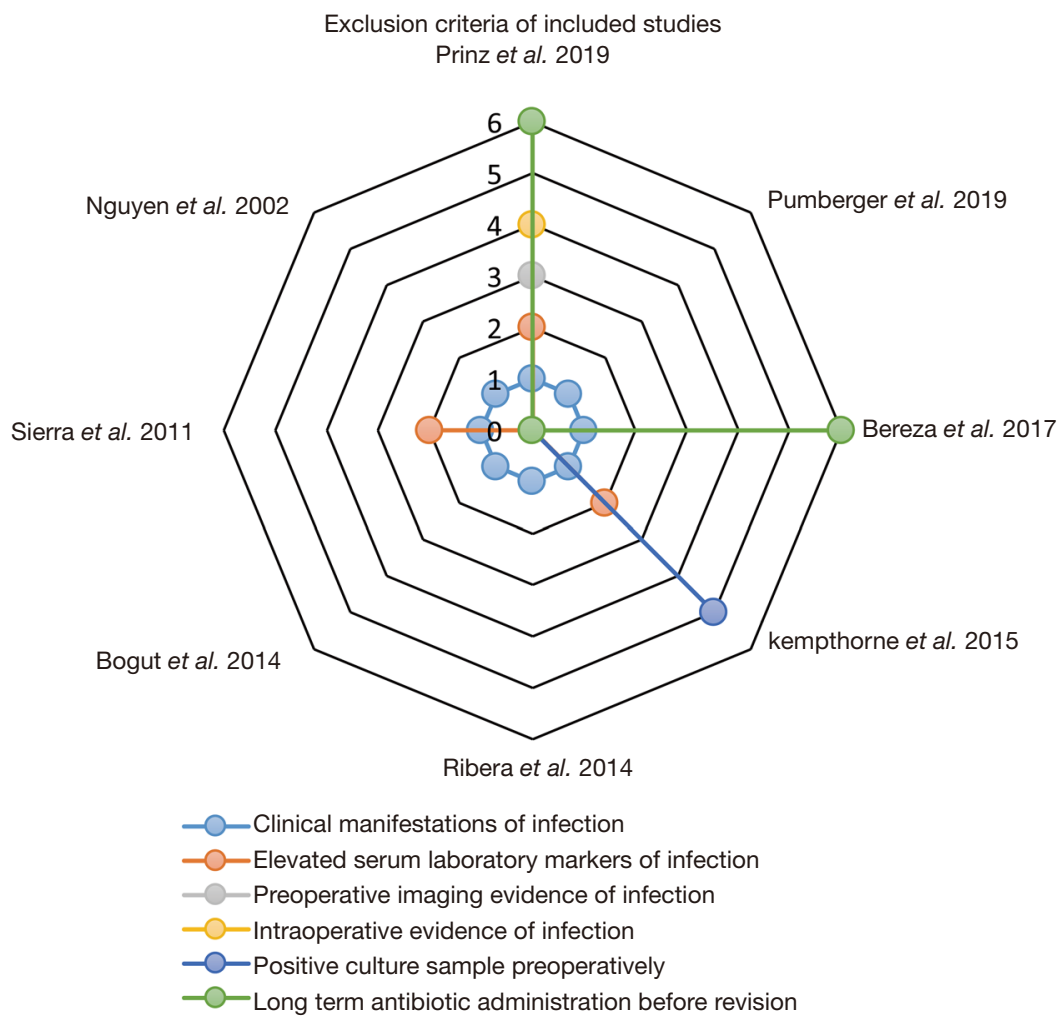

Figure 3 Patient exclusion criteria of the included studies.

(Table 2). In four $(20-22,28)$ studies, the explanted implants were vortexed ( 30 seconds to 5 minutes) before or after sonication.

\section{Quality and applicability assessment}

The QUADAS-2 results of each individual study are listed in Figure 4. The assessment showed that most studies had a low risk of bias regarding patient selection, index test, reference standard, and flow and timing. Studies that were considered to have an uncertain risk of bias with regard to patient selection included Bogut et al.'s study (28), which did not report the selection period of the included patients, and three $(21,23,28)$ studies that did not detail the imaging methods used to detect the loosening of orthopedic devices.

\section{Meta-analysis}

\section{Primary outcome}

The positive rate of sonication fluid culture ranged from $5-58 \%$ (Figure 5). Significant heterogeneity $\left(\mathrm{I}^{2}=88 \%\right)$ existed across these studies, and the pooled yield for detecting the presence of microorganisms in patients with presumed aseptic loosening was 32\% (95\% CI: 20-46\%) (Figure 6A).

In two $(22,28)$ studies involving a total of 91 patients, the yield of aspirated fluid culture was reported. There was no significant heterogeneity $\left(\mathrm{I}^{2}=0 \%\right)$ found between these studies, and the pooled yield for detecting presence of microorganisms was 13\% (95\% CI: 7-21\%) (Figure 6B).

In three $(22,26,28)$ studies involving a total of 180 patients, the yield of periprosthetic soft tissue culture was reported. Significant heterogeneity $\left(I^{2}=87 \%\right)$ existed between these studies, and the pooled yield for detecting the presence of microorganisms was $22 \%$ (95\% CI: 8-41\%) (Figure 6C).

\section{Subgroup analysis}

Subgroup analysis was first conducted according to the type of orthopedic devices with presumed aseptic loosening. Two $(20,21)$ studies involving a total of 114 patients reported the yield of sonication fluid culture for detecting 


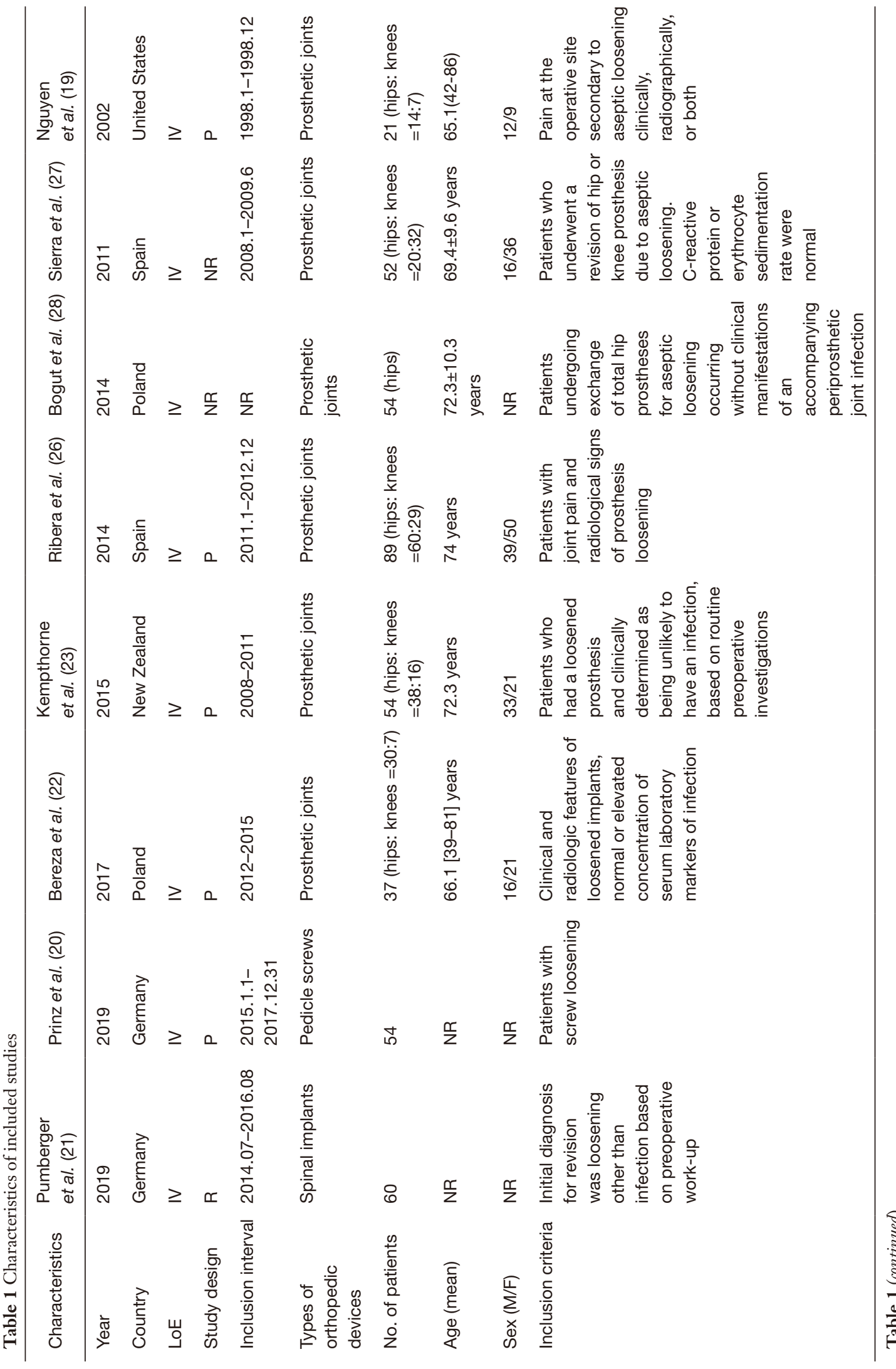




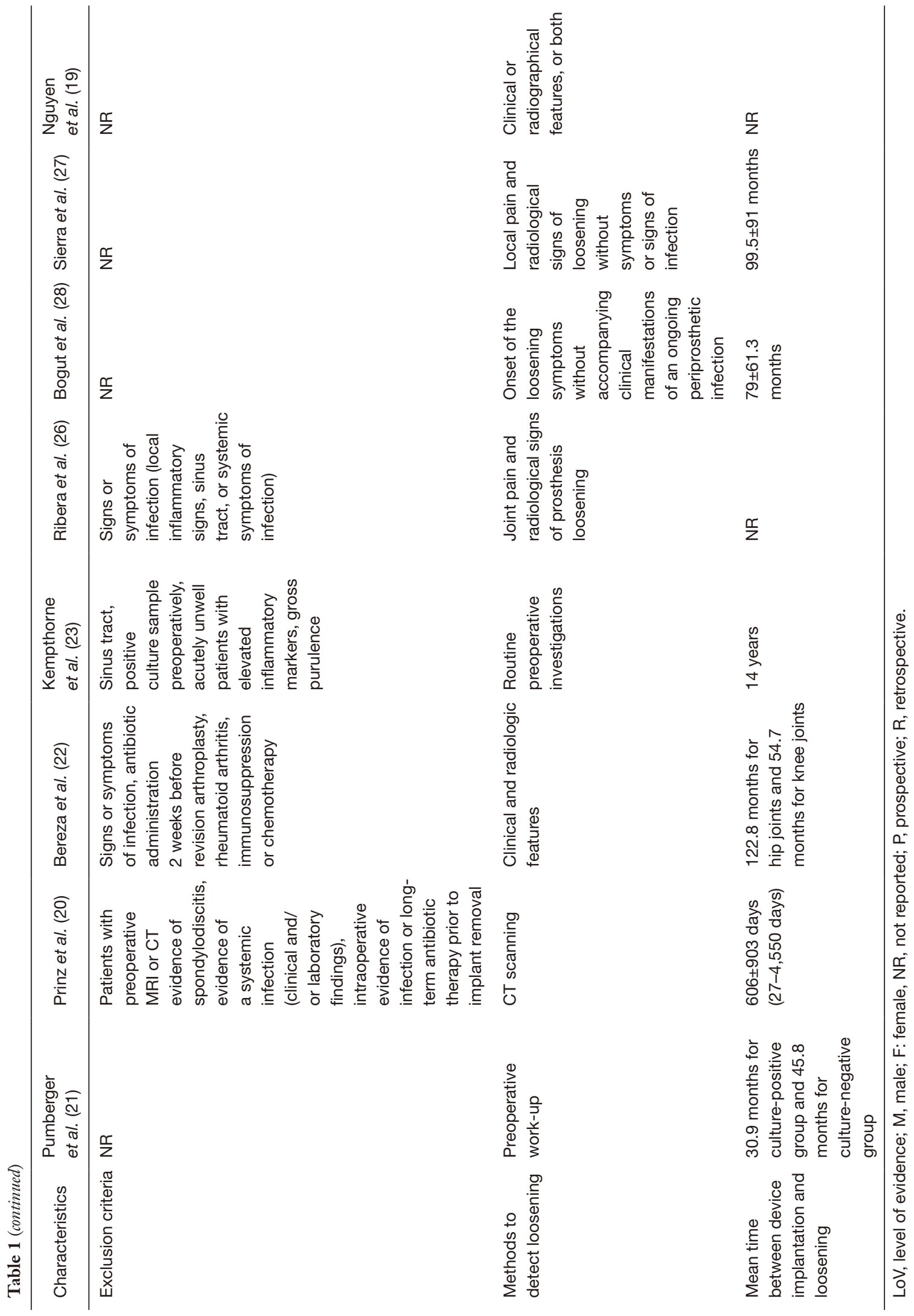


Table 2 Parameter and results of sonication culture in included studies

\begin{tabular}{|c|c|c|c|c|c|c|c|c|}
\hline Author & Year & $\begin{array}{l}\text { Duration of } \\
\text { sonication } \\
\text { procedure }\end{array}$ & Frequency & Vortexing & $\begin{array}{l}\text { Comparison } \\
\text { with PTC }\end{array}$ & $\begin{array}{l}\text { Comparison } \\
\text { with AFC }\end{array}$ & Microbiology of SFC & $\begin{array}{l}\text { Quantification } \\
\text { of bacteria }\end{array}$ \\
\hline \multirow{2}{*}{$\begin{array}{l}\text { Pumberger } \\
\text { et al. (21) }\end{array}$} & \multirow{2}{*}{2019} & \multirow{2}{*}{$3 \mathrm{~min}$} & \multirow{2}{*}{ NR } & \multirow{2}{*}{$\begin{array}{l}\text { Yes ( } 30 \mathrm{~s} \text { prior } \\
\text { to sonication) }\end{array}$} & \multirow{2}{*}{ No } & \multirow{2}{*}{ No } & P. acnes $(\mathrm{n}=9)$ & \multirow{2}{*}{ Yes } \\
\hline & & & & & & & S. aureus $(n=2)$ & \\
\hline \multirow[t]{4}{*}{ Prinz et al. (20) } & \multirow[t]{4}{*}{2019} & \multirow[t]{4}{*}{$1 \mathrm{~min}$} & \multirow[t]{4}{*}{$40 \mathrm{kHz}$} & \multirow{4}{*}{$\begin{array}{l}\text { Yes ( } 30 \mathrm{~s} \text { prior } \\
\text { to sonication } \\
\text { and } 30 \mathrm{~s} \text { after } \\
\text { sonication) }\end{array}$} & \multirow[t]{4}{*}{ No } & \multirow[t]{4}{*}{ No } & CoNS $(n=15)$ & \multirow[t]{4}{*}{ Yes } \\
\hline & & & & & & & $P$. acnes $(\mathrm{n}=6)$ & \\
\hline & & & & & & & Enterococcus & \\
\hline & & & & & & & faecalis $(n=1)$ & \\
\hline \multirow{4}{*}{$\begin{array}{l}\text { Bereza } \\
\text { et al. (22) }\end{array}$} & \multirow{4}{*}{2017} & \multirow{4}{*}{$5 \mathrm{~min}$} & \multirow{4}{*}{$40 \mathrm{kHz}$} & \multirow{4}{*}{$\begin{array}{l}\text { Yes (5 min/ } \\
2,500 \mathrm{rpm} \text { after } \\
\text { sonication) }\end{array}$} & \multirow{4}{*}{ Yes } & \multirow{4}{*}{ Yes } & R. pickettii $(\mathrm{n}=4)$ & \multirow{4}{*}{ Yes } \\
\hline & & & & & & & S. aureus $(n=2)$ & \\
\hline & & & & & & & E. cloacae $(\mathrm{n}=2)$ & \\
\hline & & & & & & & Others $(n=4)$ & \\
\hline \multirow{2}{*}{$\begin{array}{l}\text { Kempthorne } \\
\text { et al. (23) }\end{array}$} & \multirow[t]{2}{*}{2015} & \multirow[t]{2}{*}{$10 \mathrm{~min}$} & \multirow[t]{2}{*}{$67 \mathrm{kHz}$} & \multirow[t]{2}{*}{ NR } & \multirow[t]{2}{*}{ NR } & NR & CoNS $(n=6)$ & Yes \\
\hline & & & & & & & S. aureus $(n=2)$ & \\
\hline Ribera et al. (26) & 2014 & $5 \mathrm{~min}$ & $40 \mathrm{kHz}$ & NR & Yes & NR & CoNS $(n=45)$ & Yes \\
\hline & & & & $\begin{array}{l}\text { to sonication } \\
\text { and } 30 \mathrm{~s} \text { after }\end{array}$ & & & S. aureus $(n=1)$ & \\
\hline & & & & sonication) & & & P. acnes $(\mathrm{n}=1)$ & \\
\hline & & & & & & & $\begin{array}{l}\text { Enterobacter cloacae } \\
(\mathrm{n}=1)\end{array}$ & \\
\hline Sierra et al. (27) & 2011 & $5 \mathrm{~min}$ & $40 \mathrm{kHz}$ & NR & NR & NR & CoNS $(n=44)$ & Yes \\
\hline & & & & & & & $\begin{array}{l}\text { Micrococcus luteus } \\
(\mathrm{n}=2)\end{array}$ & \\
\hline & & & & & & & $\begin{array}{l}\text { Streptococcus spp. } \\
(n=2)\end{array}$ & \\
\hline $\begin{array}{l}\text { Nguyen } \\
\text { et al. (19) }\end{array}$ & 2002 & $30 \mathrm{~min}$ & $60 \mathrm{kHz}$ & NR & NR & NR & CoNS $(n=1)$ & Yes \\
\hline
\end{tabular}

a , polymicrobial growth is included. NR, not reported; PTC, periprosthetic tissue culture; AFC, aspirated fluid culture; SFC, sonication fluid culture; CoNS, coagulase-negative staphylococcus. 


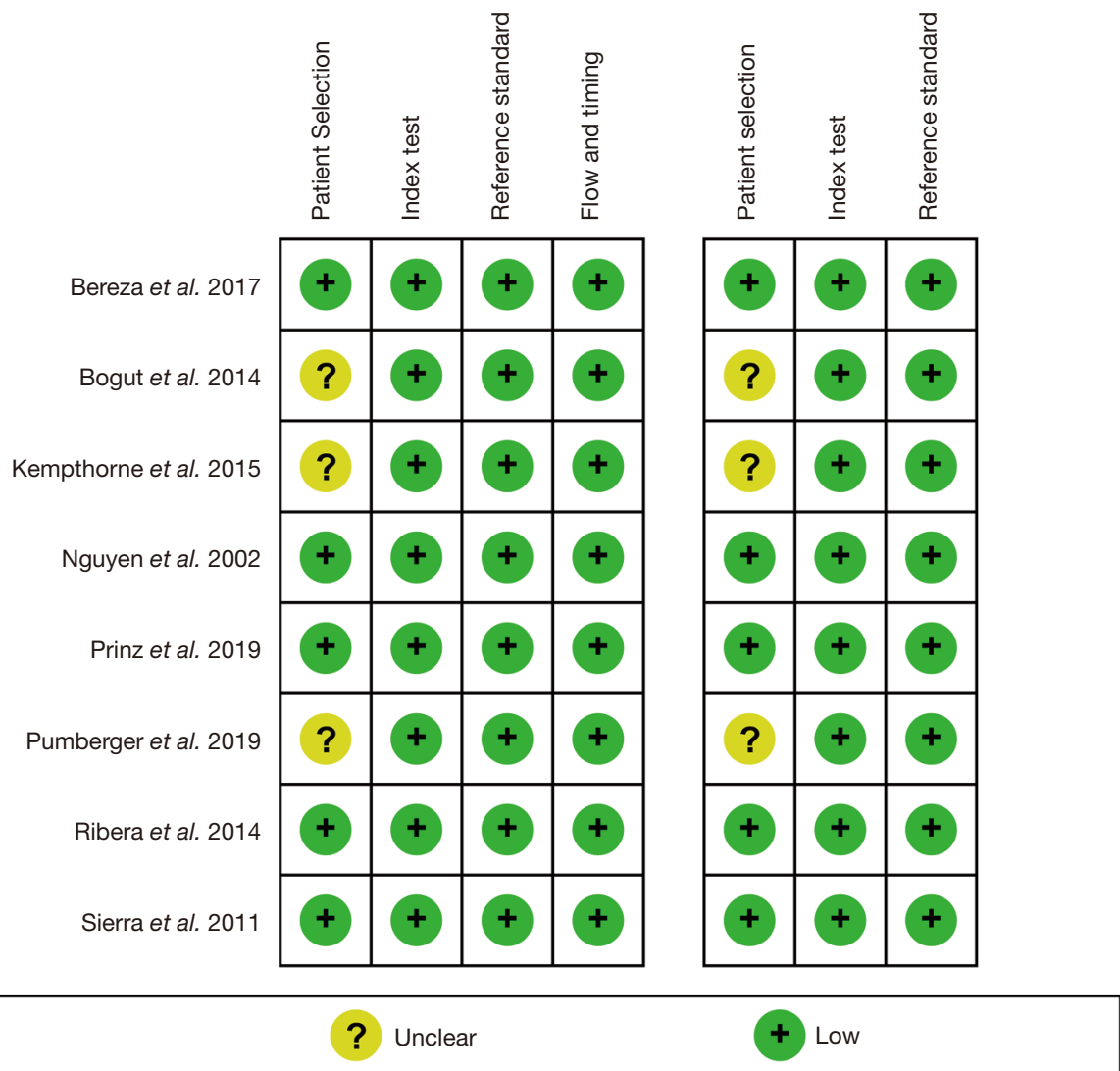

Figure 4 Methodological quality summary of the included studies. Red indicates a high risk of bias, yellow an unclear risk of bias and green a low risk of bias.

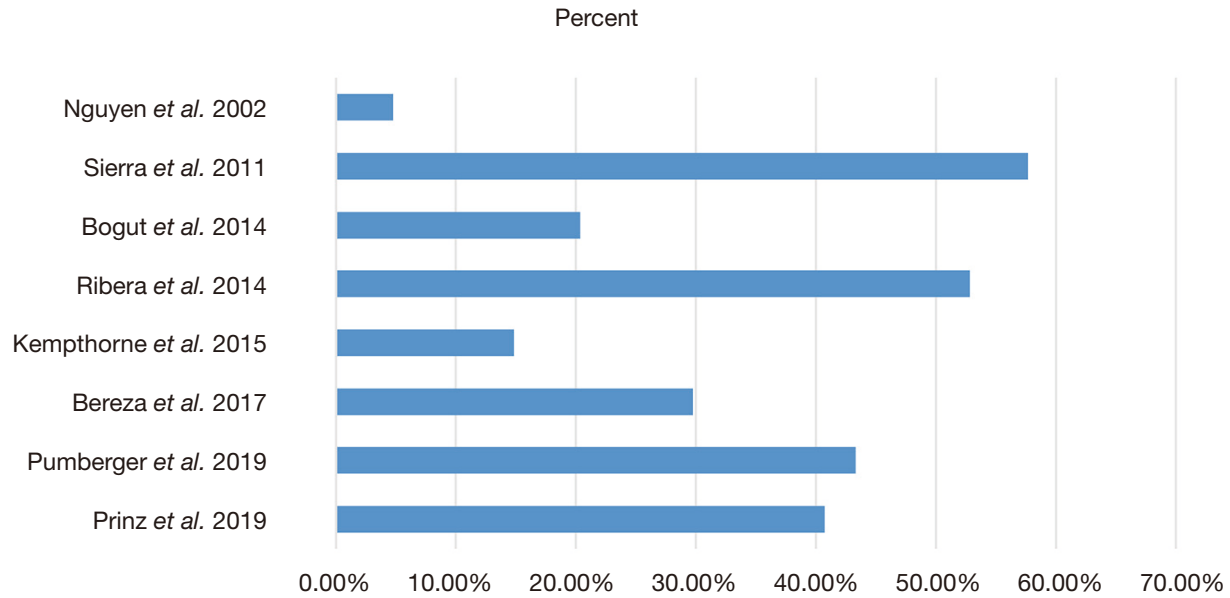

Figure 5 Percentage of cases with positive sonication culture in the included studies. 
A

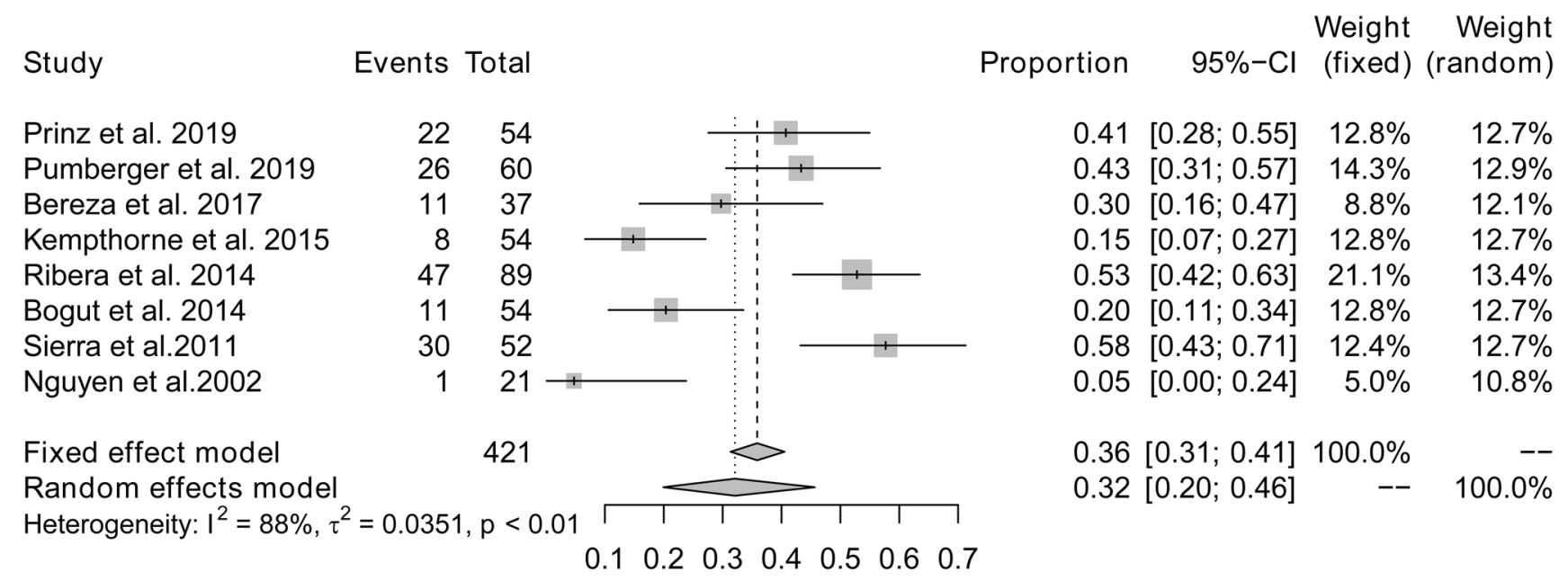

B

Study

Bereza et al. 2017

Bogut et al. 2014

Fixed effect model

Random effects model

Heterogeneity: $I^{2}=0 \%, \tau^{2}=0, p=0.48$

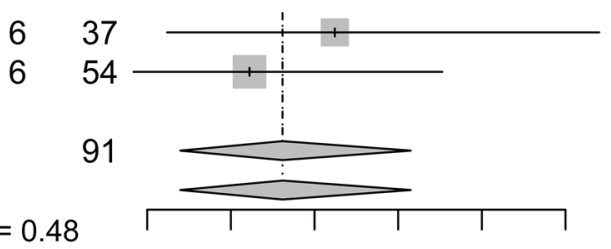

$\begin{array}{llllll}0.05 & 0.1 & 0.15 & 0.2 & 0.25 & 0.3\end{array}$

C

Study

Events Total

Bereza et al. 2017

Ribera et al. 2014

Bogut et al. 2014

Fixed effect model

Random effects model

Heterogeneity: $I^{2}=87 \%, \tau^{2}=0.0297, p<0.01$

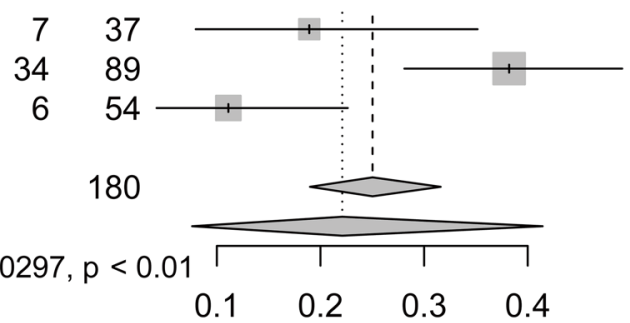

Weight Weight

Proportion $\quad 95 \%-\mathrm{Cl}$ (fixed) (random)

$0.16[0.06 ; 0.32] \quad 40.7 \% \quad 40.7 \%$

$0.11[0.04 ; 0.23] \quad 59.3 \% \quad 59.3 \%$

$0.13[0.07 ; 0.21] 100.0 \% \quad--$

$0.13[0.07 ; 0.21] \quad-\quad 100.0 \%$

Figure 6 Forest plots of the overall yield of sonication fluid culture (A), aspirated fluid culture (B) and periprosthetic soft tissue culture (C), with corresponding $95 \%$ confidence intervals.

microorganisms from spinal fusion instrumentations. No significant heterogeneity $\left(\mathrm{I}^{2}=0 \%\right)$ was found between these studies, and the pooled yield was $42 \%$ (95\% CI: $33-51 \%)$ (Figure $7 A)$. A further $6(19,22,23,26-28)$ studies involving a total of 307 patients reported the yield of sonication fluid culture in artificial joints. Significant heterogeneity $\left(\mathrm{I}^{2}=91 \%\right)$ was observed across these studies, and the pooled yield for detecting the presence of microorganisms was 29\% (95\% CI: $13-47 \%$ ) (Figure 7B).

A subgroup analysis was then conducted according to the duration of sonication and vortexing. In $4(20,21,26,27)$ studies, the total duration of sonication and vortexing was $\leq 5$ minutes, and the pooled yield was $49 \%$ (95\% CI: $41-56 \%)$ (Figure $8 A)$. No significant heterogeneity $\left(\mathrm{I}^{2}=31 \%\right)$ 
A

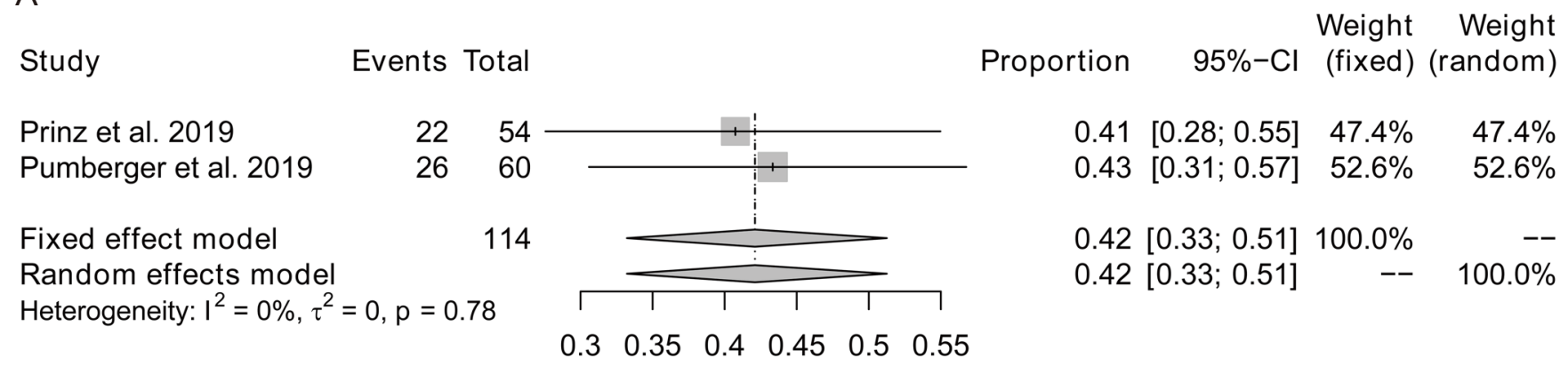

B

Study Events Total

Proportion $\quad 95 \%-\mathrm{Cl}$ (fixed) (random)

Bereza et al. 2017

Kempthorne et al. 2015

Ribera et al. 2014

Bogut et al. 2014

Sierra et al.2011

Nguyen et al.2002

$\begin{array}{lll}11 & 37\end{array}$

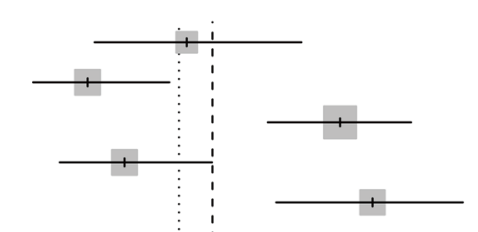

$0.30[0.16 ; 0.47] \quad 12.1 \% \quad 16.4 \%$

$0.15[0.07 ; 0.27] \quad 17.6 \% \quad 17.0 \%$

$0.53[0.42 ; 0.63] \quad 29.0 \% \quad 17.6 \%$

$0.20[0.11 ; 0.34] \quad 17.6 \% \quad 17.0 \%$

$0.58[0.43 ; 0.71] \quad 16.9 \% \quad 17.0 \%$

$0.05[0.00 ; 0.24] \quad 6.8 \% \quad 15.0 \%$

Fixed effect model

Random effects model

Heterogeneity: $I^{2}=91 \%, \tau^{2}=0.0507, p<0.01$

307

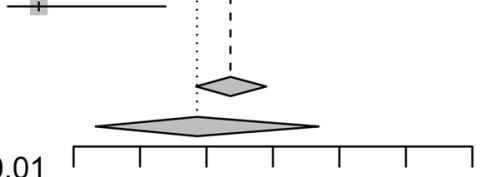

$\begin{array}{lllllll}0.1 & 0.2 & 0.3 & 0.4 & 0.5 & 0.6 & 0.7\end{array}$
$0.34[0.28 ; 0.39] 100.0 \%$

$0.29[0.13 ; 0.47] \quad--\quad 100.0 \%$

Figure 7 Forest plots of the overall yield of sonication fluid culture for spinal instruments (A) and artificial joints (B) with corresponding $95 \%$ confidence intervals.

was observed across these studies. For studies $(22,23,28)$ with a total sonication and vortexing duration of $>5$ minutes and $\leq 10$ minutes, the pooled yield was $21 \%$ (95\% CI: $13-$ $29 \% ; \mathrm{I}^{2}=31 \%$ ) (Figure 8B). Nguyen et al. sonicated explanted endoprostheses for 30 minutes, with which the yield of sonication fluid culture was only 5\% (19). This indicated that the significant heterogeneity across the included studies was mainly attributable to differences in the duration of sonication and vortexing. Furthermore, with the extension of the duration of sonication and vortexing, the positive rate of sonication fluid culture gradually decreased.

\section{Secondary outcome}

All studies reported the proportion of coagulasenegative staphylococcus (CoNS) colonization in positive cases of sonication fluid culture, and the pooled proportion was $74 \%$ (95\% CI: 51-92\%). Significant heterogeneity was found between the studies $\left(\mathrm{I}^{2}=89 \%\right)$ (Figure 9).

\section{Qualitative analysis}

\section{Spectrum of causal pathogens}

Several studies indicated that multiple isolates could be found in the same patient, with CoNS being the bacteria commonly identified in cultures from artificial joints and spinal fusion instrumentations (20-23). In a study involving 44 patients, Sierra et al. reported that CoNS was isolated in all positive cases of sonication fluid culture (27). In two $(20,21)$ studies concerning spinal fusion instrumentations, Cutibacterium acnes (C. acnes) was also found; however, this pathogen was not reported as being present in six studies $(19,22,23,26-28)$ which evaluated sonication fluid culture yields in artificial joints.

PCR of sonicated fluid of explanted orthopedic devices Bereza et al. (29) used PCR amplification of $16 \mathrm{~S}$ ribosomal RNA, the component of a $30 \mathrm{~S}$ small subunit of a prokaryotic ribosome, to examine sonication fluid 


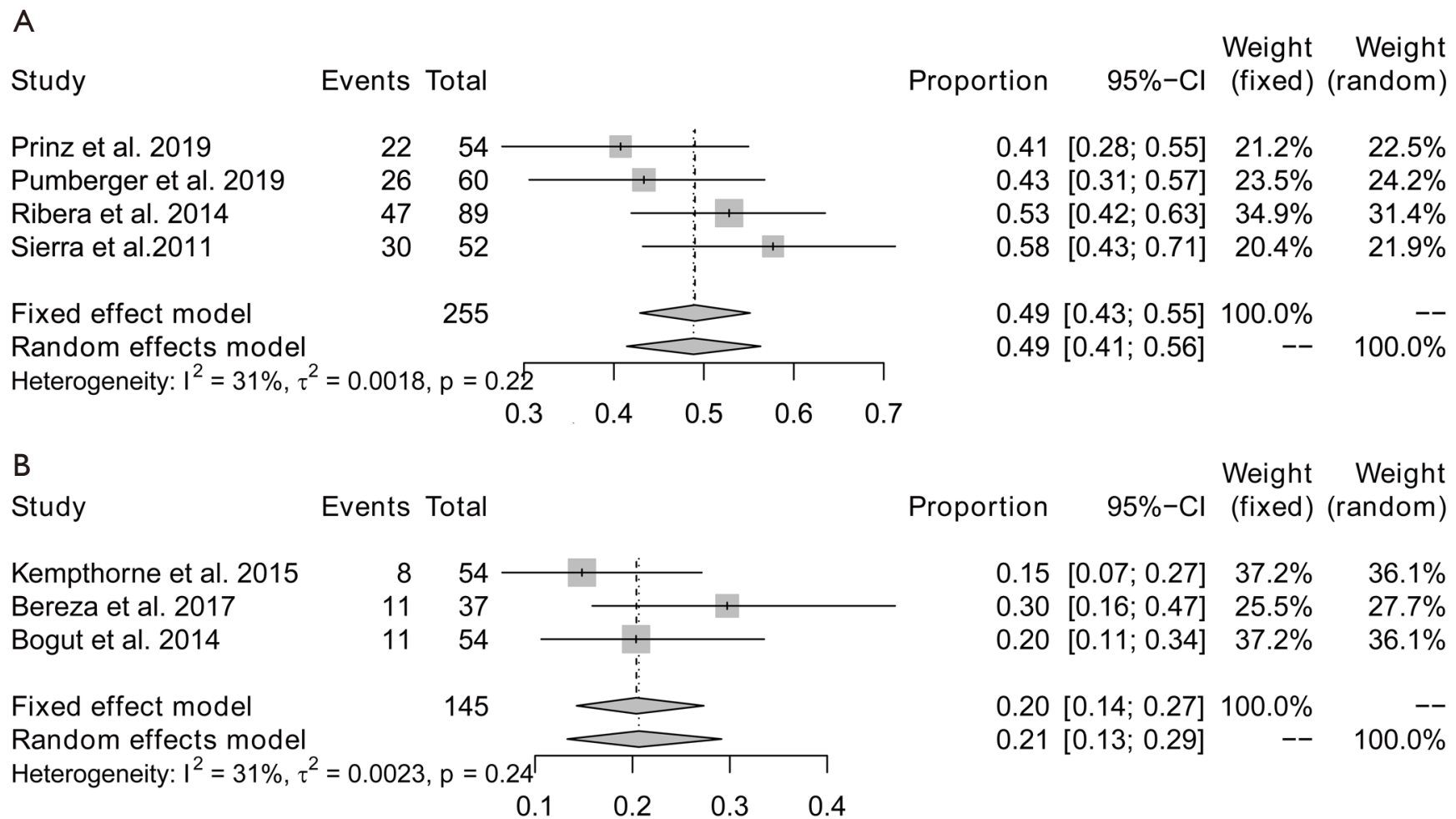

Figure 8 Forest plots of the overall yields of studies with a total duration of sonication and vortexing not exceeding 5 minutes (A), and those with a duration longer than 5 minutes but not exceeding 10 minutes (B), with corresponding $95 \%$ confidence intervals.

Study

Prinz et al. 2019

Pumberger et al. 2019

Bereza et al. 2017

Kempthorne et al. 2015

Ribera et al. 2014

Bogut et al. 2014

Sierra et al.2011

Nguyen et al.2002

Fixed effect model

Random effects model

Heterogeneity: $I^{2}=89 \%, \tau^{2}=0.1022, p<0.01$

Events Total

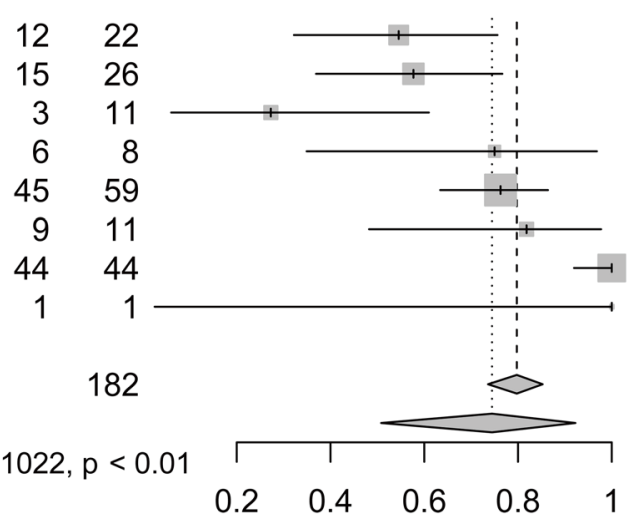

Weight Weight

Proportion $\quad 95 \%-\mathrm{Cl}$ (fixed) (random)

$0.55[0.32 ; 0.76] \quad 12.1 \% \quad 14.0 \%$

$0.58[0.37 ; 0.77] \quad 14.3 \% \quad 14.3 \%$

$0.27[0.06 ; 0.61] \quad 6.0 \% \quad 12.8 \%$

$0.75[0.35 ; 0.97] \quad 4.4 \% \quad 11.9 \%$

$0.76[0.63 ; 0.86] \quad 32.4 \% \quad 15.0 \%$

$0.82[0.48 ; 0.98] \quad 6.0 \% \quad 12.8 \%$

$1.00[0.92 ; 1.00] \quad 24.2 \% \quad 14.8 \%$

$1.00[0.03 ; 1.00] \quad 0.5 \% \quad 4.5 \%$

$0.80[0.74 ; 0.85] 100.0 \% \quad--$

$0.74[0.51 ; 0.92] \quad--\quad 100.0 \%$

Figure 9 Forest plots of the pooled proportion of coagulase-negative staphylococcus (CoNS) colonization in positive case of sonication fluid culture with corresponding $95 \%$ confidence intervals. 
Table 3 Outcome of revision surgery

\begin{tabular}{|c|c|c|c|c|c|c|c|}
\hline $\begin{array}{l}\text { Outcome of } \\
\text { sonication fluid }\end{array}$ & Subtypes & \multicolumn{2}{|c|}{ Bereza et al. (22) } & \multicolumn{2}{|c|}{ Kempthorne et al. (23) } & \multicolumn{2}{|c|}{ Nguyen et al. (19) } \\
\hline \multirow[t]{2}{*}{ 1-stage $(n)$} & Success & 5 & 16 & 8 & 45 & 1 & 20 \\
\hline & Failure & 1 & 3 & 0 & 0 & 0 & 0 \\
\hline 2-stage (n) & Success & 3 & 5 & NA & NA & NA & NA \\
\hline
\end{tabular}

P, positive; N, negative; NA, not applicable.

when microorganisms growth was not observed in either intraoperative periprosthetic soft tissue culture or in sonication fluid culture in patients with presumed aseptic loosening of artificial joints. Surprisingly, 9 out of 10 (90\%) patients showed positive microbiological results.

\section{Interrelation between sonication result and outcome of revision surgery}

The short- or long-term outcomes of revision surgery were reported in three $(19,22,23)$ studies (Table 3). Kempthorne et al. (23) applied one-stage revisions to 8 cases with positive microbiological outcomes of sonication fluid culture and 45 cases with negative outcomes. All patients eventually recovered without recurrent infection or repeated loosening of the implants. Similar results of one-stage revision were obtained by Nguyen et al. (19) and Bereza et al. (22). Bereza et al. (22) also evaluated patients with two-stage revision. Eventually, failures, characterized by prolonged antibiotic therapy or incision healing across an average of 2 years of follow-up, was found to be more frequent in the positive sonication fluid culture group $(2 / 5,40 \%)$ than in the negative sonication fluid culture groups (2/7, 28.57\%); however, the difference was not statistically significant $(\mathrm{c} 2=0.1714, \mathrm{P}=0.68)$.

\section{Discussion}

Unrecognized or subclinical infection has been implicated in contributing to aseptic loosening of orthopedic devices. In the current study, the pooled results suggest that bacterial colonization is extremely common in patients with suspected aseptic loosening of orthopedic devices and might play a greater role in the onset of this clinical complication than had been previously thought. Sonication fluid culture, which has already been widely used in the diagnosis of PJI, produced a positive yield rate in $32 \%$ of patients with presumed aseptic loosening of orthopedic devices, which is superior to that of periprosthetic soft tissue culture and aspirated joint fluid culture.

Contamination during the initial surgery, hematogenous dissemination and contiguous spread have been described as common pathways by which bacteria reach the surface of implants and consequently form biofilm $(30,31)$. Due to the paucity of blood vessels, immune cells function inefficiently on orthopedic devices and other foreign bodies (32). Zimmerli et al. (33) demonstrated that the bactericidal and phagocytic capacity of neutrophile granulocytes was significantly compromised by the presence of foreign bodies, which was very highly to favor bacterial colonization. In addition to host factors, the virulence of colonizing pathogens also influences the development of implant-associated infections. Unlike acute infections, which are mostly induced by Staphylococcus aureus (34), the bacteria identified by sonication fluid culture in the current study were mainly low-virulent microorganisms, such as CoNS and $C$. acnes. These bacteria are capable of colonizing in vivo for a long time without inducing overt clinical manifestations. CoNS was found to be the most common causative agents of PJIs (30-43\%), and there was no clear distinction between bacterial colonization and subclinical ORI (28). Inflammatory laboratory parameters, such as white blood cell count, erythrocyte sedimentation rate, and C-reactive protein, might not be significantly increased in cases with presumed aseptic loosening of orthopedic devices; however, this does not rule out the possibility of bacterial colonization in biofilm as previously stated (27). Moreover, although bacterial colonization does not necessarily lead to implant-associated infection, it may induce local subclinical chronic inflammatory processes and osteolysis, which may result in chronic loosening of the orthopedic device (35).

Subgroup analysis revealed that the significant heterogeneity seen across the included studies mainly 
resulted from differences in the duration of sonication and vortexing. A total duration of more than 5 minutes will significantly decrease the yield of sonication fluid culture. This may be related to the reduced viability or even destruction of detached bacteria. The use of an ultrasound bath for 1-5 minutes at $40 \mathrm{kHz}$ may yield the optimal diagnostic performance for bacterial colonization (49\%, 95\% CI: 41-56\%). Different microorganisms and infection risks were also observed to be associated with different types of orthopedic devices and procedures. There was a higher proportion of subclinical infection or bacterial colonization in spinal fusion instrumentations with presumed aseptic loosening than that found in artificial joints. This might be due to the increased risk of intraoperative contamination resulting from the relatively longer duration of spinal surgery, and the deeper location of spinal instrumentations, which makes it harder for the symptoms of infection to manifest, in comparison with arthroplasty. Another reason for this may pertain to the duration between initial and revision surgery in spinal and arthroplasty patients. Spinal surgery components, such as pedicle screws, usually lose function slowly, bringing a gradual onset of symptoms, whereas only a small loosening of arthroplasty components will cause manifest clinical symptoms (22). Therefore, the time in between initial and revision surgeries in spinal implant patients is generally longer, allowing for greater bacterial colonization (22). Finally, certain differences existed regarding the constitution of identified pathogens colonizing on artificial joints and spinal instruments, albeit all being represented by low-virulent microorganisms. $C$. acnes, which are commonly detected in presumed aseptic loosening of spinal fusion instruments other than artificial joints, was a common cause of late-presenting infection after spinal surgery (21).

The use of sonication fluid culture to specifically investigate presumed aseptic loosening of osteo-synthetic materials such as steel plates, screws, and K-wires, has not been widely reported. However, positive bacterial yields using sonication fluid culture have been observed in osteo-synthetic materials with no clinical evidence of infection. In 2019, Fuchs et al. (36) reported on the removal of 203 osteo-synthetic implants around the knee and hip for related soft tissue irritations or before elective total joint arthroplasty. Sonication fluid cultures revealed positive yields in $27 \%$ of implants (54/203), with the most frequently identified microorganism being CoNS (46\%). Another study conducted by Knabl et al. (37) demonstrated a similar bacterial colonization rate $(56.1 \%)$ of osteosynthetic implants in patients for whom PCR amplification of sonication fluid showed no clinical or laboratory signs of infection. These results confirm that the presence of bacteria in cases with no clinical signs of infection is a relevant issue for who undergo receiving implantation of orthopedic devices.

In this study, the pooled data revealed that other methods used for detecting bacterial colonization or subclinical infection, such as peri-implant fibrotic tissue culture and aspirated fluid culture, were less effective in leading to the identification of microorganisms (22\% and $13 \%$, respectively) in comparison with sonication fluid culture. Leitner et al. (4) included 110 patients who underwent revision surgery following open spinal fusion with metal explantation to the thoracic and lumbar spine. They found that the detection power of wound swabs taken from a loosened screw hole $(43.6 \%)$ was inferior to that of sonication fluid culture $(71.9 \%)$. The higher positive rate of sonication fluid culture may be related to the fact that this method allows bacteria from the whole implant surface to be examined, while wound swab, tissue culture, and aspirated fluid culture can only examine a limited area of the implants. The extremely high positive rate and detection efficiency of PCR of sonicated fluid reported in some studies may render a suspicion of contamination $(22,29)$. Moreover, the presence of bacterial $16 \mathrm{~S}$ ribosomal RNA material on PCR merely indicates the presence of bacterial genetic material, rather than the existence of active or indolent bacterial infection. Technical sophistication and the relative substantial costs of sonication PCR of orthopedic devices may also limit its wider applicability $(29,37)$.

Distinguishing aseptic loosening from PJI is important, as the treatment of the two groups is significantly different. Revision surgery for the presumed aseptic loosening of orthopedic devices with positive and negative microbiologic results of sonication fluid culture demonstrated similarly satisfactory short- and long-term prognoses based on limited evidence $(19,22,23)$. After implant removal, the potential source of infection cannot be identified; thus, soft tissue recovery may occur in those patients with evidence of implant colonization. Some authors have proposed the application of antibiotics coinciding with the antibiogram for 4 to 6 weeks (4); however, this is still under debate.

There are multiple limitations of this study that merit consideration. Firstly, the definition of presumed aseptic 
loosening across the included studies was somewhat subjective, which means that different inclusion and exclusion criteria may have been adopted. This may partially explain the significant heterogeneity in the quantitative analysis. Secondly, it must be stated that bacterial implant colonization may not lead to the loosening of orthopedic devices or associated infection. The question remains whether loosening is caused by the presence of organisms or whether infection is facilitated by the environment of loosening. Thirdly, the number of included studies is small and underpowered, which could hinder generalizability of the conclusion. Finally, the reported potential of sonication procedure-based contamination could bring bias.

\section{Conclusions}

Colonization by low-virulent microorganisms may exist in many patients with clinically presumed aseptic loosening of orthopedic devices. A sonication bath of explanted orthopedic devices for $1-5$ minutes at $4 \mathrm{kHz}$ could act as a powerful diagnostic tool to detect bacterial colonization. However, organism colonization detected by sonication fluid culture may not influence the outcome of one-stage revision surgery for presumed aseptic loosening. More research is required to determine whether sonication fluid culture should be incorporated into the routine treatment strategy for orthopedic device loosening.

\section{Acknowledgments}

Funding: This work was supported by the Young Taishan Scholars Program of Shandong Province (No. tsqn201909183) and the Academic promotion programme of Shandong First Medical University (2020RC008) to Q Zhang.

\section{Footnote}

Reporting Checklist: The authors have completed the PRISMA reporting checklist. Available at http://dx.doi. org/10.21037/apm-20-1228

Peer Review File: Available at http://dx.doi.org/10.21037/ apm-20-1228

Conflicts of Interest: All authors have completed the ICMJE uniform disclosure form (available at http://dx.doi. org/10.21037/apm-20-1228). The authors have no conflicts of interest to declare.

Ethical Statement: The authors are accountable for all aspects of the work in ensuring that questions related to the accuracy or integrity of any part of the work are appropriately investigated and resolved.

Open Access Statement: This is an Open Access article distributed in accordance with the Creative Commons Attribution-NonCommercial-NoDerivs 4.0 International License (CC BY-NC-ND 4.0), which permits the noncommercial replication and distribution of the article with the strict proviso that no changes or edits are made and the original work is properly cited (including links to both the formal publication through the relevant DOI and the license). See: https://creativecommons.org/licenses/by-nc-nd/4.0/.

\section{References}

1. Galbusera F, Volkheimer D, Reitmaier S, et al. Pedicle screw loosening: a clinically relevant complication? Eur Spine J 2015;24:1005-16.

2. Yokoe DS, Avery TR, Platt R, et al. Reporting surgical site infections following total hip and knee arthroplasty: impact of limiting surveillance to the operative hospital. Clin Infect Dis 2013;57:1282-8.

3. Koh CK, Zeng I, Ravi S, et al. Periprosthetic Joint Infection Is the Main Cause of Failure for Modern Knee Arthroplasty: An Analysis of 11,134 Knees. Clin Orthop Relat Res 2017;475:2194-201.

4. Leitner L, Malaj I, Sadoghi P, et al. Pedicle screw loosening is correlated to chronic subclinical deep implant infection: a retrospective database analysis. Eur Spine J 2018;27:2529-35.

5. Costerton JW, Stewart PS, Greenberg EP. Bacterial biofilms: a common cause of persistent infections. Science 1999;284:1318-22.

6. Trampuz A, Piper KE, Jacobson MJ, et al. Sonication of removed hip and knee prostheses for diagnosis of infection. N Engl J Med 2007;357:654-63.

7. Arciola CR, Campoccia D, Speziale P, et al. Biofilm formation in Staphylococcus implant infections. A review of molecular mechanisms and implications for biofilmresistant materials. Biomaterials 2012;33:5967-82.

8. Coraça-Huber DC, Fille M, Hausdorfer J, et al. Staphylococcus aureus biofilm formation and antibiotic susceptibility tests on polystyrene and metal surfaces. J Appl Microbiol 2012;112:1235-43. 
9. Mauffrey C, Herbert B, Young H, et al. The role of biofilm on orthopaedic implants: the "Holy Grail" of posttraumatic infection management? Eur J Trauma Emerg Surg 2016;42:411-6.

10. Zimmerli W, Trampuz A, Ochsner PE. Prosthetic-joint infections. N Engl J Med 2004;351:1645-54.

11. Del Pozo JL, Patel R. Clinical practice. Infection associated with prosthetic joints. N Engl J Med 2009;361:787-94.

12. Metsemakers WJ, Kortram K, Morgenstern M, et al. Definition of infection after fracture fixation: A systematic review of randomized controlled trials to evaluate current practice. Injury 2018;49:497-504.

13. Parvizi J, Della VC. AAOS Clinical Practice Guideline: diagnosis and treatment of periprosthetic joint infections of the hip and knee. J Am Acad Orthop Surg 2010;18:771-2.

14. Osmon DR, Berbari EF, Berendt AR, et al. Diagnosis and management of prosthetic joint infection: clinical practice guidelines by the Infectious Diseases Society of America. Clin Infect Dis 2013;56:e1-e25.

15. Parvizi J, Zmistowski B, Berbari EF, et al. New definition for periprosthetic joint infection: from the Workgroup of the Musculoskeletal Infection Society. Clin Orthop Relat Res 2011;469:2992-4.

16. Parvizi J, Gehrke T, Chen AF. Proceedings of the International Consensus on Periprosthetic Joint Infection. Bone Joint J 2013;95-B:1450-2.

17. Pitt WG, Ross SA. Ultrasound increases the rate of bacterial cell growth. Biotechnol Prog 2003;19:1038-44.

18. Liu H, Zhang Y, Li L, et al. The application of sonication in diagnosis of periprosthetic joint infection. Eur J Clin Microbiol Infect Dis 2017;36:1-9.

19. Nguyen LL, Nelson CL, Saccente M, et al. Detecting bacterial colonization of implanted orthopaedic devices by ultrasonication. Clin Orthop Relat Res 2002;403:29-37.

20. Prinz V, Bayerl S, Renz N, et al. High frequency of lowvirulent microorganisms detected by sonication of pedicle screws: a potential cause for implant failure. J Neurosurg Spine 2019:31:424-9.

21. Pumberger M, Burger J, Strube P, et al. Unexpected positive cultures in presumed aseptic revision spine surgery using sonication. Bone Joint J 2019;101-B:621-4.

22. Bereza PL, Ekiel A, Augusciak-Duma A, et al. Identification of Asymptomatic Prosthetic Joint Infection: Microbiologic and Operative Treatment Outcomes. Surg Infect (Larchmt) 2017;18:582-7.
23. Kempthorne JT, Ailabouni R, Raniga S, et al. Occult Infection in Aseptic Joint Loosening and the Diagnostic Role of Implant Sonication. Biomed Res Int 2015;2015:946215.

24. Whiting PF, Rutjes AW, Westwood ME, et al. QUADAS-2: a revised tool for the quality assessment of diagnostic accuracy studies. Ann Intern Med 2011;155:529-36.

25. Schwarzer G, Carpenter JR, Rücker G. Meta-analysis with R. Springer, 2015.

26. Ribera A, Morata L, Moranas J, et al. Clinical and microbiological findings in prosthetic joint replacement due to aseptic loosening. J Infect 2014;69:235-43.

27. Sierra JM, Garcia S, Martinez-Pastor JC, et al. Relationship between the degree of osteolysis and cultures obtained by sonication of the prostheses in patients with aseptic loosening of a hip or knee arthroplasty. Arch Orthop Trauma Surg 2011;131:1357-61.

28. Bogut A, Niedzwiadek J, Koziol-Montewka M, et al. Sonication as a diagnostic approach used to investigate the infectious etiology of prosthetic hip joint loosening. Pol J Microbiol 2014;63:299-306.

29. Bereza PL, Ekiel A, Augusciak-Duma A, et al. Identification of silent prosthetic joint infection: preliminary report of a prospective controlled study. Int Orthop 2013;37:2037-43.

30. Shohat N, Goswami K, Tan TL, et al. Increased Failure After Irrigation and Debridement for Acute Hematogenous Periprosthetic Joint Infection. J Bone Joint Surg Am 2019;101:696-703.

31. Di Benedetto P, Di Benedetto ED, Salviato D, et al. Acute periprosthetic knee infection: is there still a role for DAIR? Acta Biomed 2017;88:84-91.

32. Zimmerli W, Sendi P. Pathogenesis of implant-associated infection: the role of the host. Semin Immunopathol 2011;33:295-306.

33. Zimmerli W, Lew PD, Waldvogel FA. Pathogenesis of foreign body infection. Evidence for a local granulocyte defect. J Clin Invest 1984;73:1191-200.

34. Hassoun A, Linden PK, Friedman B. Incidence, prevalence, and management of MRSA bacteremia across patient populations-a review of recent developments in MRSA management and treatment. Crit Care 2017;21:211.

35. Rienmüller A, Borens O. Propionibacterium prosthetic joint infection: experience from a retrospective database analysis. Eur J Orthop Surg Traumatol 2016;26:429-34.

36. Fuchs M, Kinzel S, Gwinner C, et al. Clinically 
Asymptomatic Patients Show a High Bacterial

Colonization Rate of Osteosynthetic Implants Around the Knee but Not the Hip. J Arthroplasty 2019;34:1761-6.

37. Knabl L, Kuppelwieser B, Mayr A, et al. High percentage of bacterial colonization of osteosynthesis material in clinically unremarkable patients. Microbiologyopen 2019;8:e00658.

Cite this article as: Zhang Q, Xi Y, Li D, Yuan Z, Dong J. The yield of sonication fluid culture for presumed aseptic loosening of orthopedic devices: a meta-analysis. Ann Palliat Med 2021;10(2):1792-1808. doi: 10.21037/apm-20-1228 